\title{
Spontaneous Cervical Spinal Epidural Hematoma Mimicking Acute Stroke
}

\author{
Ashkan Shoamanesh, Jose R. Romero, Carlos S. Kase
}

Can J Neurol Sci. 2014; 41: 533-534

In the era of thrombolysis for acute stroke management, neurologists are required to rapidly diagnose and manage patients presenting with acute neurological deficits. An awareness of stroke mimics is necessary to avoid unnecessary administration of intravenous tissue plasminogen activator, particularly in cases where thrombolysis could lead to devastating outcomes.

We present a case of spontaneous cervical spinal epidural hematoma $(\mathrm{SEH})$ presenting with unilateral deficits mimicking acute stroke.

\section{CASE RePORT}

A 65-year-old woman with multiple vascular risk factors, without history of preceding trauma or coagulopathy, was assessed as a referral for acute stroke in the emergency department. She had been in her usual health until 1.5 hours prior to presentation when she suffered sudden neck pain, followed by collapse to the floor and right-sided hemiplegia. Her weakness partially improved en route to the hospital where examination showed severe hypertension (217/116), monoparesis (4+ proximally, 4- distally) of the right arm in a pyramidal distribution, hypesthesia of the right arm to all four primary modalities, bilateral symmetric hyperreflexia and right Babinski. There was not any contralateral sensory loss or sensory level present. The remainder of her examination was unremarkable. Computed tomography (CT) angiogram (head and neck) was performed and suggested a diagnosis of cervical spinal epidural hematoma extending from C2-C5 (Figure 1), subsequently confirmed on magnetic resonance imaging (Figure 2). In retrospect, the SEH was also apparent on non-contrast CT head, which just captured the rostral end of the hematoma at the

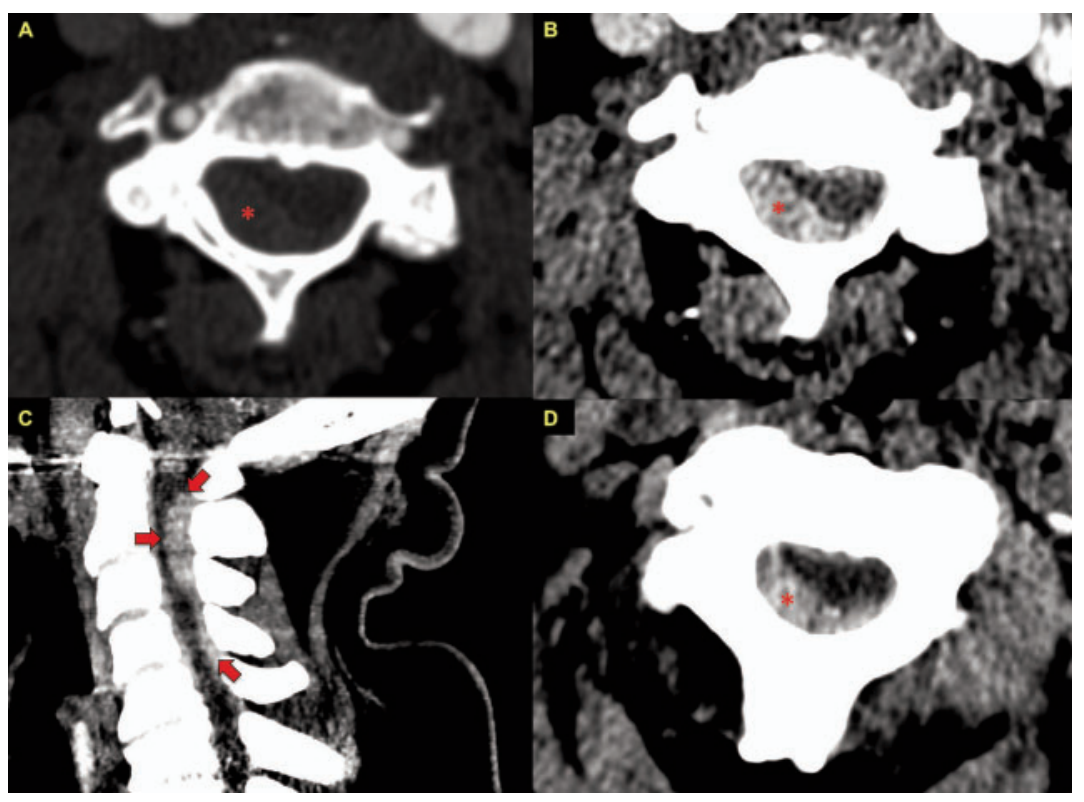

Figure 1: CT-angiogram of the neck and non-contrast $C T$ head. A predominantly right-sided posterolateral hyperdense lentiform collection from C2-C5 causing cord compression is visualized on CT-angiogram of the neck. (A) Subtle suggestion of a hyperdense lentiform collection initially visualized in real time while viewing axial images 'windowed' for visualization of the arterial system (C362/W806). (B) Window narrowing $(C 74 / W 109)$ and $(C)$ sagittal maximum intensity projections improved the conspicuity of the hematoma. (D) Most caudal slice of non-contrast CT head capturing the uppermost $C 2$ portion of the hematoma. The hematoma is marked by an asterix in the axial images $(A, B, D)$ and outlined by arrows in the sagittal images $(C)$.

\footnotetext{
From the Department of Neurology (AS), Massachusetts General Hospital, Harvard Medical School; Department of Neurology (JRR, CSK), Boston Medical Center, Boston University School of Medicine, Boston, Massachusetts, USA.

Received January 20, 2014. Final Revisions Submitted March 2, 2014.

Correspondence to: Ashkan Shoamanesh, 175 Cambridge Street, Suite 300, Boston, Massachusetts, 02114, USA. Email: ashoamanesh@mgh.harvard.edu.
} 


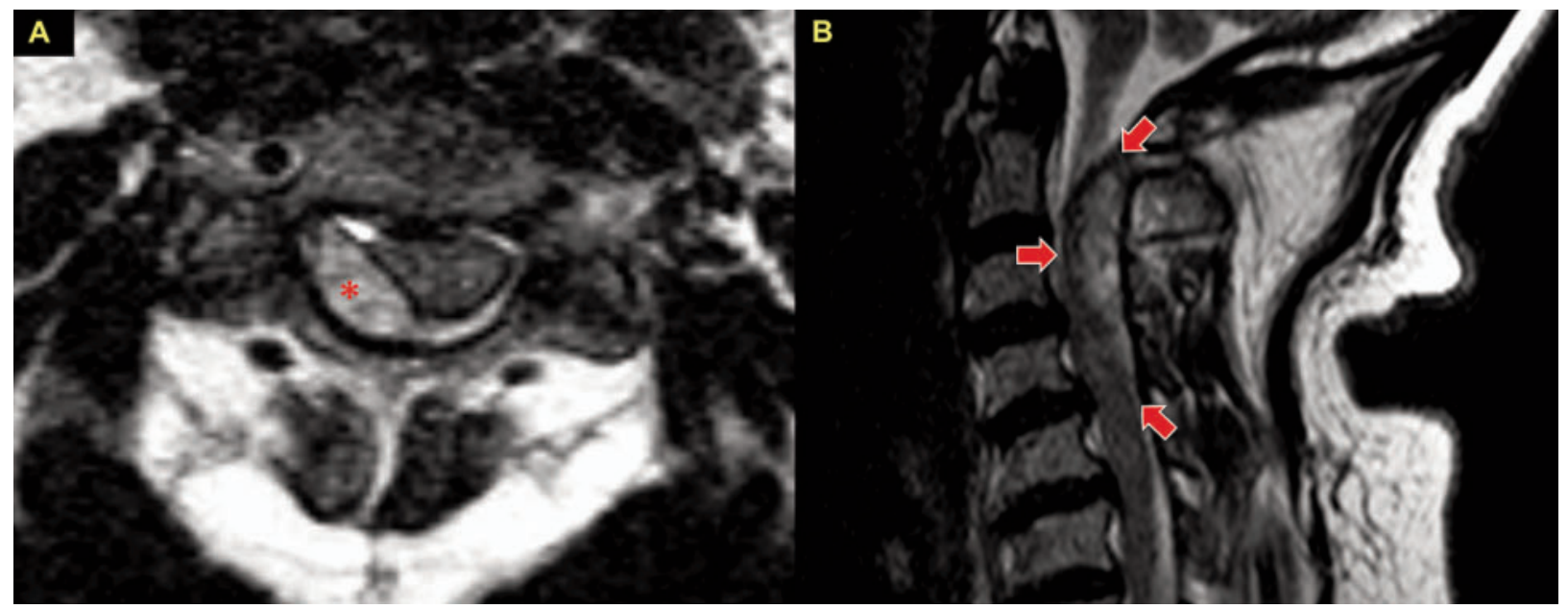

Figure 2: MRI of the cervical spine. The collection is visualized as hyperintense with a rim of hypointensity on T2-weighted MRI consistent with hemorrhage. The hematoma is marked by an asterix in the axial image (A) and outlined by arrows in the sagittal image (B).

level of C2 (Figure 1). Conventional angiogram of the cervical spine, in search of an underlying vascular anomaly, was unremarkable. The patient underwent urgent surgical evacuation of the hematoma and had made a full recovery at one month follow-up.

\section{DISCUSSION}

Spontaneous SEH is a surgical emergency with annual incidence of $0.1 / 100000^{1}$. Associated causes include underlying coagulopathy, vascular anomaly, and minor trauma/exertion, but roughly $50 \%$ of cases are idiopathic. In the setting of arterial hypertension, the source of cervical SEH may be bleeding from spinal anastomotic arteries within the epidural space, however others have argued for a venous source and that the association with hypertension is coincidental. Spinal epidural hematoma is visualized as a lentiform collection, often posterolateral, with characteristics of evolving blood products ${ }^{1}$.

Thromboembolic cerebral infarction from cervical arterial dissection is often considered in patients presenting with suddenonset neurological deficit accompanied by neck pain, and monoparesis can result from isolated cortical infarction of the pre-central gyrus. Typically SEH will present with sudden neck/back pain followed by progressive quadri/paraparesis, however rare cases with hemiparesis have been reported ${ }^{2-5}$, a number of which having received anticoagulation or thrombolysis, under the presumptive diagnosis of ischemic stroke, resulting in progression of deficits ${ }^{2,3,5}$. These reports, in addition to our own, stress that sudden-onset neck pain and absence of cranial nerve findings should raise suspicion for cervical SEH in patients with acute hemiparesis considered for thrombolysis ${ }^{3}$. We found CT-angiography of the head and neck to be useful in rapidly ruling out cervical arterial dissection, as well as intracranial arterial occlusion, suggestive of ischemic stroke, while simultaneously securing the diagnosis of cervical $\mathrm{SEH}$. Although in this particular case the hematoma extended rostral enough to also be captured on non-contrast CT head, more caudal hematomas would be missed on this modality. Accordingly, if CT-angiography is not already part of an institution's acute stroke protocol, CT-angiography including the neck should strongly be considered in such cases, with additional careful attention paid to the vertebral foramina and spinal cord while reviewing images.

\section{REFERENCES}

1. Holtås S, Heiling M, Lönntoft M. Spontaneous spinal epidural hematoma: findings at MR imaging and clinical correlation. Radiology. 1996;199(2):409-13.

2. Son S, Kang DH, Choi DS, Kim SK, Lim BH, Choi NC. A case of spontaneous spinal epidural hematoma mimicking a stroke. Neurologist. 2012;18(1):41-3.

3. Wang CC, Chang CH, Lin HJ, Lin KC, Kuo JR. Misdiagnosis of spontaneous cervical epidural haemorrhage. Eur Spine J. 2009; 18 Suppl 2:210-2.

4. Matsumoto H, Miki T, Miyaji Y, et al. Spontaneous spinal epidural hematoma with hemiparesis mimicking acute cerebral infarction: two case reports. J Spinal Cord Med. 2012;35(4): 262-6.

5. Hsieh CF, Lin HJ, Chen KT, Foo NP, Te AL. Acute spontaneous cervical spinal epidural hematoma with hemiparesis as the initial presentation. Eur J Emerg Med. 2006;13(1):36-8. 